\title{
Two Parameter Modified Ratio Estimators with Two Auxiliary Variables for Estimation of Finite Population Mean with Known Skewness, Kurtosis and Correlation Coefficient
}

Jambulingam Subramani

Pondicherry University, Puducherry, India, drjsubramani@yahoo.co.in

G Prabavathy

Pondicherry University, Puducherry, India, praba.gopal.23@gmail.com

Follow this and additional works at: http://digitalcommons.wayne.edu/jmasm

Part of the Applied Statistics Commons, Social and Behavioral Sciences Commons, and the Statistical Theory Commons

\section{Recommended Citation}

Subramani, Jambulingam and Prabavathy, G (2014) "Two Parameter Modified Ratio Estimators with Two Auxiliary Variables for Estimation of Finite Population Mean with Known Skewness, Kurtosis and Correlation Coefficient," Journal of Modern Applied Statistical Methods: Vol. 13 : Iss. 1 , Article 13. DOI: $10.22237 /$ jmasm/1398917520

Available at: http://digitalcommons.wayne.edu/jmasm/vol13/iss1/13

This Regular Article is brought to you for free and open access by the Open Access Journals at DigitalCommons@WayneState. It has been accepted for inclusion in Journal of Modern Applied Statistical Methods by an authorized editor of DigitalCommons@WayneState. 


\section{Two Parameter Modified Ratio Estimators with Two Auxiliary Variables for Estimation of Finite Population Mean with Known Skewness, Kurtosis and Correlation Coefficient}

\author{
Jambulingam Subramani \\ Pondicherry University \\ Puducherry, India
}

\author{
G. Prabavathy \\ Pondicherry University \\ Puducherry, India
}

Consider the two parameter modified ratio estimators for the estimation of finite population mean using the skewness, kurtosis and correlation coefficient of two auxiliary variables. The efficiencies of the proposed modified ratio estimators are assessed with that of the simple random sampling without replacement (SRSWOR) sample mean and some of the existing ratio estimators in terms of mean squared errors. The entire above is explained with the help of certain natural populations available in the literature.

Keywords: $\quad$ Mean squared error; natural populations; percentage relative efficiency; simple random sampling

\section{Introduction}

In survey sampling, consider the problem of estimating the population mean $\bar{Y}=\frac{1}{N} \sum_{i=1}^{N} Y_{i}$ for a finite population $U=\left\{U_{1}, U_{2}, \ldots, U_{\mathrm{N}}\right\}$ of $N$ distinct and identifiable units, where the value $Y_{i}$ is measured on $U_{i}, i=1,2,3, \ldots, N$. Normally the population mean is estimated by the sample mean obtained from a random sample of size $n$ drawn by simple random sampling without replacement (SRSWOR) from a finite population, when there is no auxiliary information available. Suppose that there is an auxiliary variable $X$ available that is positively correlated with a study variable $Y$, in this case, either a ratio estimator or linear regression estimator may be used to improve the efficiency of the SRSWOR

Dr. Subramani is an Associate Professor in the Department of Statistics. Email him at: drjsubramani@yahoo.co.in.G.Prabavathy is a Ph.D. Scholar in the Department of Statistics.Email her at: praba.gopal.23@gmail.com. 


\section{TWO PARAMETER MODIFIED RATIO ESTIMATORS}

sample mean under certain conditions (see, Cochran (1977) and Murthy (1967) for example). Further improvements can be achieved on the ratio estimator by using known parameters such as skewness, kurtosis, quartiles and coefficient of variation of the auxiliary variable; the resulting estimators are called modified ratio estimators. For further details on the modified ratio estimators, readers are referred to Kadilar and Cingi (2004, 2009), Singh and Tailor (2003, 2005), Singh (2003), Sisodia and Dwivedi (1981), Subramani (2013), Subramani and Kumarapandiyan (2012a, b, c, 2013), Upadhyaya and Singh (1999), and Yan and Tian (2010).

If two auxiliary variables exist, then several modified ratio estimators have been proposed by linking together ratio estimators, product estimators and regression estimators in order to obtain more efficient estimators. For more detailed discussion about ratio estimators and their modifications using two auxiliary variables readers are referred to: Abu-Dayyeh et al. (2003), Bandyopadhyay (1980), Cochran (1940), Kadilar and Cingi (2004, 2005), Khare et al. (2013), Murthy (1967), Naik and Gupta (1991), Olkin (1958), Perri (2004, 2007), Rao and Mudholkar (1967), Raj (1965), Sahoo and Swain (1980), Singh (2003), Singh (1965, 1967), Singh and Tailor (2003, 2005), Srivenkataramana (1980), Srivenkataramana and Tracy (1981), Tailor et al. (2011), and Tracy et al. (1996).

\section{Existing Estimators with and without auxiliary variables}

If $\left(y_{1}, y_{2}, \ldots, y_{n}\right)$ is a random sample of size $n$ drawn from a population of size $N$ using SRSWOR, then the population mean $\bar{Y}$ can be estimated by the sample mean $\bar{y}=\frac{1}{n} \sum_{i=1}^{n} y_{i}$, which is an unbiased estimator, and its variance is given by:

$$
V(\overline{\mathrm{y}})=\frac{(1-f)}{n} S_{y}^{2}, \text { where } S_{y}^{2}=\frac{1}{(N-1)} \sum_{i=1}^{N}\left(Y_{i}-\bar{Y}\right)^{2}, f=\frac{n}{N}
$$

The ratio estimator for estimating the population mean $\bar{Y}$ of the study variable $Y$ is defined as

$$
\hat{\bar{Y}}_{R}=\frac{\bar{y}}{\bar{x}} \bar{X}=\hat{R} \bar{X}
$$

The mean squared error of the ratio estimator $\hat{\bar{Y}}_{R}$ to the first degree of approximation is: 


\section{SUBRAMANI \& PRABAVATHY}

$$
\operatorname{MSE}\left(\hat{\bar{Y}}_{R}\right)=\frac{(1-f)}{n} \bar{Y}^{2}\left(C_{y}^{2}+C_{X}^{2}-2 p_{x y} C_{x} C_{y}\right) .
$$

Singh (2003) suggested a ratio estimator with two auxiliary variables for estimating a population mean:

$$
\hat{\overline{Y_{1}}}=\bar{y}\left(\frac{\bar{X}_{1}}{x_{1}}\right)\left(\frac{\bar{X}_{2}}{x_{2}}\right)
$$

The mean squared error of $\hat{\bar{Y}}_{1}$ to the first order of approximation is:

$$
\operatorname{MSE}(\hat{\bar{Y}})=\frac{(1-f)}{n} \bar{Y}^{2}\left(C_{y}^{2}+C_{X_{1}}^{2}+C_{X_{2}}^{2}-2 p_{y x_{1}} C_{x_{2}} C_{y}+2 p_{x_{1} x_{2}} C_{x_{1}} C_{x_{2}}\right)
$$

Singh and Tailor (2005) suggested the following modified ratio cum product estimator with known correlation coefficient between auxiliary variables:

$$
\hat{\bar{Y}}=\bar{y}\left(\frac{\bar{X}_{1}+p_{x_{1} x_{2}}}{\bar{x}_{1}+p_{x_{1} x_{2}}}\right)\left(\frac{\bar{x}_{2}+p_{x_{1} x_{2}}}{\bar{X}_{2}+p_{x_{1} x_{2}}}\right) .
$$

The mean squared error of $\hat{\bar{Y}}_{2}$ to the first order of approximation is:

$$
\operatorname{MSE}\left(\hat{\bar{Y}}_{2}\right)=\frac{1-f}{n} \bar{Y}^{2}\left[C_{y}^{2}+\mu_{1}^{*} C_{x_{1}}^{2}\left(\mu_{1}^{*}-2 K_{y x_{1}}\right)+\mu_{2}^{*} C_{x_{2}}^{2}\left(\mu_{2}^{*}+2\left(K_{y x_{2}}-\mu_{1}^{*} K_{x_{1} x_{2}}\right)\right)\right]
$$

where

$$
K_{y x_{1}}=\rho_{y x_{1}} \frac{C_{y}}{C_{x_{1}}}, K_{y x_{2}}=\rho_{y x_{2}} \frac{C_{y}}{C_{x_{2}}}, K_{x_{1} y_{2}}=\rho_{x_{1} y_{2}} \frac{C_{x_{1}}}{C_{x_{2}}}, \mu_{1}^{*}=\frac{\bar{X}_{1}}{\bar{X}_{1}+\rho_{x x_{1} x_{2}}} \text { and } \mu_{2}^{*}=\frac{\bar{X}_{2}}{\bar{X}_{2}+\rho_{x_{1} x_{2}}}
$$

and $\rho_{x_{1} x_{2}}$ is the coefficient of correlation between $X_{1}$ and $X_{2}$.

Kadilar and Cingi (2005) proposed a new ratio estimator using two auxiliary variables as: 


\section{TWO PARAMETER MODIFIED RATIO ESTIMATORS}

$$
\hat{\bar{Y}}_{3}=\bar{y}\left(\frac{\bar{X}_{1}}{\bar{x}_{1}}\right)^{\alpha_{1}}\left(\frac{\bar{X}_{2}}{\bar{x}_{2}}\right)^{\alpha_{2}}+b_{1}\left(\bar{X}_{1}-\bar{x}_{1}\right)+b_{2}\left(\bar{X}_{2}-\bar{x}_{2}\right) .
$$

The mean squared error of $\bar{Y}_{3}$ to the first order of approximation is:

$$
\operatorname{MSE}\left(\hat{\bar{Y}}_{3}\right) \cong \frac{1-f}{n}\left\{\begin{array}{l}
S_{y}^{2}+\left(\alpha_{1} R_{1}+B_{1}\right)^{2} S_{x_{1}}^{2} \\
+\left(\alpha_{2} R_{2}+B_{2}\right)^{2} S_{x_{1}}^{2} \\
-2\left(\alpha_{1} R_{1}+B_{1}\right) S_{y x_{1}} \\
-2\left(\alpha_{2} R_{2}+B_{2}\right) S_{y x_{2}} \\
+2\left(\alpha_{1} R_{1}+B_{1}\right)\left(\alpha_{2} R_{2}+B_{2}\right) S_{x_{1} x_{2}}
\end{array}\right\}
$$

where $B_{1}=\frac{S_{x y}}{S_{x_{1}}^{2}}, B_{2}=\frac{S_{x y}}{S_{x_{2}}^{2}}, R_{1}=\frac{\bar{Y}}{\bar{X}_{1}}$ and $R_{2}=\frac{\bar{Y}}{\bar{X}_{2}}$.

Perri (2007) suggested some modified ratio cum product estimators using two auxiliary variables for estimating the population mean:

$$
\hat{\bar{Y}}_{4}=\bar{y} \frac{\hat{t}_{2}}{\hat{t}_{1}} \frac{\bar{X}_{1}}{\bar{X}_{2}}, \hat{\bar{Y}}_{5}=\bar{y} \frac{\bar{X}_{1}}{\hat{t}_{1}} \frac{\bar{X}_{2}}{\hat{t}_{2}} \text { and } \hat{\bar{Y}}_{6}=\bar{y} \frac{\hat{t}_{1}}{\hat{t}_{2}} \frac{\bar{X}_{2}}{\bar{X}_{1}}
$$

where $\hat{t}_{1}=\bar{x}_{1}+\alpha_{1}\left(\bar{X}_{1}-\bar{x}_{1}\right)$ and $\hat{t}_{2}=\bar{x}_{2}+\alpha_{2}\left(\bar{X}_{2}-\bar{x}_{2}\right)$.

The mean squared errors of $\hat{\bar{Y}}_{4}, \hat{\bar{Y}}_{5}, \hat{\bar{Y}}_{6}$ to the first order of approximation are:

$$
\begin{aligned}
& \operatorname{MSE}\left(\hat{\bar{Y}}_{4}\right)=\frac{1-f}{n}\left[S_{y}^{2}+\gamma_{x_{1}}^{2}+\gamma_{x_{2}}^{2}-2\left(\gamma_{y x_{1}}-\gamma_{y x_{2}}+\gamma_{x_{1} x_{2}}\right)\right] \\
& \operatorname{MSE}\left(\hat{\bar{Y}}_{5}\right)=\frac{1-f}{n}\left[S_{y}^{2}+\gamma_{x_{1}}^{2}+\gamma_{x_{2}}^{2}-2\left(\gamma_{y x_{1}}+\gamma_{y x_{2}}-\gamma_{x_{1} x_{2}}\right)\right] \\
& \operatorname{MSE}\left(\hat{\bar{Y}}_{6}\right)=\frac{1-f}{n}\left[S_{y}^{2}+\gamma_{x_{1}}^{2}+\gamma_{x_{2}}^{2}+2\left(\gamma_{y x_{1}}-\gamma_{y x_{2}}-\gamma_{x_{1} x_{2}}\right)\right]
\end{aligned}
$$




\section{SUBRAMANI \& PRABAVATHY}

where $\gamma_{x_{1} x_{2}}=\left(1-\alpha_{1}\right)\left(1-\alpha_{2}\right) R_{1} R_{2} S_{x_{1} x_{2}}, \quad \gamma_{x_{1}}=\left(1-\alpha_{1}\right) R_{1} S_{x_{1}}, \quad \gamma_{x_{2}}=\left(1-\alpha_{2}\right) R_{2} S_{x_{2}}$ $\gamma_{y x_{1}}=\left(1-\alpha_{1}\right) R_{1} S_{y x_{1}}$. and $\gamma_{y x_{2}}=\left(1-\alpha_{2}\right) R_{2} S_{y x_{2}}$.

This article is concerned with estimating the population mean of a study variable $Y$ by two parameter modified ratio estimators with known correlation coefficient, skewness and kurtosis of two auxiliary variables $X_{1}$ and $X_{2}$.

\section{Proposed Two Parameter Modified Ratio Estimators}

Whenever one or two auxiliary variables exist, a number of estimators including ratio, regression, product and chain ratio type estimators and their linear combinations have been proposed in the literature. These estimators are improved by using the known values of parameters such as skewness, kurtosis and coefficient of variation of the auxiliary variables. All of these estimators are functions of the ratio, product, regression estimators and their linear combinations; hence, an attempt is made herein to introduce the weighted average of the ratio estimators whenever there are two auxiliary variables available. As a result, two parameter modified ratio estimators with known correlation coefficient, skewness, kurtosis and their linear combinations of two auxiliary variables are proposed.

When the coefficient of kurtosis $\beta_{2}\left(X_{1}\right)$ of the auxiliary variable $X_{1}$, and $\beta_{2}\left(X_{2}\right)$ of the auxiliary variable $X_{2}$ is known, the following two parameter modified ratio estimator is proposed:

$$
\hat{\bar{Y}}_{S P 1}=\bar{y}\left(\frac{\alpha_{1}\left[\bar{X}_{1}+\beta_{2}\left(X_{1}\right)\right]+\alpha_{2}\left[\bar{X}_{2}+\beta_{2}\left(X_{2}\right)\right]}{\alpha_{1}\left[\bar{x}_{1}+\beta_{2}\left(X_{1}\right)\right]+\alpha_{2}\left[\bar{x}_{2}+\beta_{2}\left(X_{2}\right)\right]}\right) \text {. }
$$

Using the linear combinations of coefficient of kurtosis $\beta_{2}\left(X_{1}\right)$ of the auxiliary variable $X_{1}, \beta_{2}\left(X_{2}\right)$ of the auxiliary variable $X_{2}$ and correlation coefficient $\rho_{x_{1} X_{2}}$ between $X_{1}$ and $X_{2}$, the following two parameter modified ratio estimators are proposed:

$$
\hat{\bar{Y}}_{S P 2}=\bar{y}\left(\frac{\alpha_{1}\left[\rho_{x_{1} x_{2}} \bar{X}_{1}+\beta_{2}\left(X_{1}\right)\right]+\alpha_{2}\left[\rho_{x_{1} x_{2}} \bar{X}_{2}+\beta_{2}\left(X_{2}\right)\right]}{\alpha_{1}\left[\rho_{x_{1} x_{2}} \bar{x}_{1}+\beta_{2}\left(X_{1}\right)\right]+\alpha_{2}\left[\rho_{x_{1} x_{2}} \bar{x}_{2}+\beta_{2}\left(X_{2}\right)\right]}\right)
$$

and 


\section{TWO PARAMETER MODIFIED RATIO ESTIMATORS}

$$
\hat{\bar{Y}}_{S P 3}=\bar{y}\left(\frac{\alpha_{1}\left[\beta_{2}\left(X_{1}\right) \bar{X}_{1}+\rho_{x_{1} x_{2}}\right]+\alpha_{2}\left[\beta_{2}\left(X_{2}\right) \bar{X}_{2}+\rho_{x_{1} x_{2}}\right]}{\alpha_{1}\left[\beta_{2}\left(X_{1}\right) \bar{x}_{1}+\rho_{x_{1} x_{2}}\right]+\alpha_{2}\left[\beta_{2}\left(X_{2}\right) \bar{x}_{2}+\rho_{x_{1} x_{2}}\right]}\right) .
$$

Using the linear combinations of coefficient of skewness $\beta_{1}\left(X_{1}\right)$ of the auxiliary variable $X_{1}, \beta_{1}\left(X_{2}\right)$ of the auxiliary variable $X_{2}$, coefficient of kurtosis $\beta_{2}$ $\left(X_{1}\right)$ of the auxiliary variable $X_{1}$ and $\beta_{2}\left(X_{2}\right)$ of the auxiliary variable $X_{2}$ the following two parameter modified ratio estimators are proposed:

$$
\hat{\bar{Y}}_{S P 4}=\bar{y}\left(\frac{\alpha_{1}\left[\beta_{2}\left(X_{1}\right) \bar{X}_{1}+\beta_{1}\left(X_{1}\right)\right]+\alpha_{2}\left[\beta_{2}\left(X_{2}\right) \bar{X}_{2}+\beta_{1}\left(X_{2}\right)\right]}{\alpha_{1}\left[\beta_{2}\left(X_{1}\right) \bar{x}_{1}+\beta_{1}\left(X_{1}\right)\right]+\alpha_{2}\left[\beta_{2}\left(X_{2}\right) \bar{x}_{2}+\beta_{1}\left(X_{2}\right)\right]}\right)
$$

and

$$
\hat{\bar{Y}}_{S P 5}=\bar{y}\left(\frac{\alpha_{1}\left[\beta_{1}\left(X_{1}\right) \bar{X}_{1}+\beta_{2}\left(X_{1}\right)\right]+\alpha_{2}\left[\beta_{1}\left(X_{2}\right) \bar{X}_{2}+\beta_{2}\left(X_{2}\right)\right]}{\alpha_{1}\left[\beta_{1}\left(X_{1}\right) \bar{x}_{1}+\beta_{2}\left(X_{1}\right)\right]+\alpha_{2}\left[\beta_{1}\left(X_{2}\right) \bar{x}_{2}+\beta_{2}\left(X_{2}\right)\right]}\right) .
$$

In general, the estimators proposed in (14) to (18) can be defined as particular cases of the estimator:

$$
\hat{\bar{Y}}_{S P T}=\bar{y}\left(\frac{\alpha_{1}\left[\bar{X}_{1}+T_{1}\right]+\alpha_{2}\left[\bar{X}_{2}+T_{2}\right]}{\alpha_{1}\left[\bar{x}_{1}+T_{1}\right]+\alpha_{2}\left[\bar{x}_{2}+T_{2}\right]}\right) .
$$

For suitable choices of $T_{1}$ and $T_{2}$ in (19), the estimators defined in (14) to (18) are obtained.

Suppose that,

i. if $T_{1}=\beta_{2}\left(X_{1}\right)$ and $T_{2}=\beta_{2}\left(X_{2}\right)$ in (19), then $\hat{\bar{Y}}_{S P T}$ becomes $\hat{\bar{Y}}_{S P 1}$ as defined in (14);

ii. if $T_{1}=\frac{\beta_{2}\left(X_{1}\right)}{\rho_{x_{1} x_{2}}}$ and $T_{2}=\frac{\beta_{2}\left(X_{2}\right)}{\rho_{x_{1} x_{2}}}$ in (19), then $\hat{\bar{Y}}_{S P T}$ becomes $\hat{\bar{Y}}_{S P 2}$ as defined in (15);

iii. if $T_{1}=\frac{\rho_{x_{1} x_{2}}}{\beta_{2}\left(X_{1}\right)}$ and $T_{2}=\frac{\rho_{x_{1} x_{2}}}{\beta_{2}\left(X_{2}\right)}$ in (19), then $\hat{\bar{Y}}_{S P T}$ becomes $\hat{\bar{Y}}_{S P 3}$ as defined in (16); 


\section{SUBRAMANI \& PRABAVATHY}

iv. if $T_{1}=\frac{\beta_{1}\left(X_{1}\right)}{\beta_{2}\left(X_{1}\right)}$ and $T_{2}=\frac{\beta_{1}\left(X_{2}\right)}{\beta_{2}\left(X_{2}\right)}$ in (19), then $\hat{\bar{Y}}_{S P T}$ becomes $\hat{\bar{Y}}_{S P 4}$ as defined in (17); and

v. if $T_{1}=\frac{\beta_{2}\left(X_{1}\right)}{\beta_{1}\left(X_{1}\right)}$ and $T_{2}=\frac{\beta_{2}\left(X_{2}\right)}{\beta_{1}\left(X_{2}\right)}$ in (19), then $\hat{\bar{Y}}_{S P T}$ becomes $\hat{\bar{Y}}_{S P 5}$ as defined in (18).

\section{Derivation of Mean Squared Error of the proposed estimators}

The mean squared error of the proposed estimator $\hat{\bar{Y}}_{S P T}$ is derived as follows. If $e_{0}=\frac{\bar{y}-\bar{Y}}{\bar{Y}}, e_{1}=\frac{\bar{x}_{1}-\bar{X}_{1}}{\bar{X}_{1}}$, and $e_{2}=\frac{\bar{x}_{2}-\bar{X}_{2}}{\bar{X}_{2}}$, then $\bar{y}=\bar{Y}\left(1+e_{0}\right), \quad \bar{x}_{1}=\bar{X}_{1}\left(1+e_{1}\right)$, and $\overline{\mathrm{x}}_{2}=\bar{X}_{2}\left(1+e_{2}\right)$. From the definition of $e_{0}$ and $e_{1}, E\left[e_{0}\right]=E\left[e_{1}\right]=0$ is obtained where $E\left[e_{0}^{2}\right]=\frac{(1-f)}{n} C_{y}^{2}, \quad E\left[e_{1}^{2}\right]=\frac{1-f}{n} C_{x_{1}}^{2}, \quad E\left[e_{2}^{2}\right]=\frac{1-f}{n} C_{x_{2}}^{2}$, $E\left(e_{0} e_{1}\right)=\frac{1-f}{n} \rho_{y x_{1}} C_{y} C_{x_{1}}, E\left(e_{0} e_{2}\right)=\frac{1-f}{n} \rho_{y x_{2}} C_{y} C_{x_{2}}$ and $E\left(e_{1} e_{2}\right)=\frac{1-f}{n} \rho_{x_{1} x_{2}} C_{x_{1}} C_{x_{2}}$. The proposed estimator $\hat{\bar{Y}}_{S P T}$ can be written in terms of $e_{0}, e_{1}$ and $e_{2}$ as:

$$
\begin{aligned}
& \hat{\bar{Y}}_{S P T}=\bar{Y}\left(1+e_{0}\right)\left(\frac{\alpha_{1}\left[\bar{X}_{1}+T_{1}\right]+\alpha_{2}\left[\bar{X}_{2}+T_{2}\right]}{\alpha_{1}\left[\bar{X}_{1}\left(1+e_{1}\right)+T_{1}\right]+\alpha_{2}\left[\bar{X}_{2}\left(1+e_{2}\right)+T_{2}\right]}\right) \\
& \Rightarrow \hat{\bar{Y}}_{S P T}=\bar{Y}\left(1+e_{0}\right)\left(\frac{\alpha_{1} \bar{X}_{1}+\alpha_{2} \bar{X}_{2}+\alpha_{1} T_{1}+\alpha_{2} T_{2}}{\alpha_{1} \bar{X}_{1}+\alpha_{2} \bar{X}_{2}+\alpha_{1} T_{1}+\alpha_{2} T_{2}+\alpha_{1} \bar{X}_{1} e_{1}+\alpha_{2} \bar{X}_{2} e_{2}}\right) \\
& \Rightarrow \hat{\bar{Y}}_{S P T}=\bar{Y}\left(1+e_{0}\right)\left(\frac{1}{1+\theta_{1}^{\prime} e_{1}+\theta_{2}^{\prime} e_{2}}\right), \theta_{1}^{\prime}=\frac{\alpha_{1} \bar{X}_{1}}{\alpha_{1}\left[\bar{X}_{1}+T_{1}\right]+\alpha_{2}\left[\bar{X}_{2}+T_{2}\right]} \text { and } \theta_{2}^{\prime}=\frac{\alpha_{2} \bar{X}_{2}}{\alpha_{1}\left[\bar{X}_{1}+T_{1}\right]+\alpha_{2}\left[\bar{X}_{2}+T_{2}\right]} \\
& \Rightarrow \hat{\bar{Y}}_{S P T}=\bar{Y}\left(1+e_{0}\right)\left(1+\theta_{1}^{\prime} e_{1}+\theta_{2}^{\prime} e_{2}\right)^{-1} \\
& \Rightarrow \hat{\bar{Y}}_{S P T}=\bar{Y}\left(1+e_{0}\right)\left(1-\theta_{1}^{\prime} e_{1}-\theta_{2}^{\prime} e_{2}+\left(\theta_{1}^{\prime} e_{1}+\theta_{2}^{\prime} e_{2}\right)^{2}\right) \\
& \Rightarrow \hat{\bar{Y}}_{S P T}=\bar{Y}\left(1+e_{0}\right)\left(1-\theta_{1}^{\prime} e_{1}-\theta_{2}^{\prime} e_{2}+\theta_{1}^{\prime 2} e_{1}^{2}+\theta_{2}^{\prime 2} e_{2}^{2}+2 \theta_{1}^{\prime} e_{1} \theta_{2}^{\prime} e_{2}\right)
\end{aligned}
$$




\section{TWO PARAMETER MODIFIED RATIO ESTIMATORS}

Neglecting higher order terms

$\hat{\bar{Y}}_{S P T}-Y=\bar{Y} e_{0}-\bar{Y} \theta_{1}^{\prime} e_{1}-\bar{Y} \theta_{2}^{\prime} e_{2}+\bar{Y} \theta_{1}^{\prime} e_{1} \theta_{2}^{\prime} e_{2}-\bar{Y} \theta_{1}^{\prime} e_{0} e_{1}-\bar{Y} \theta_{2}^{\prime} e_{0} e_{2}$

and squaring and taking expectations on both sides results in:

$$
\begin{aligned}
& \operatorname{MSE}\left(\hat{\bar{Y}}_{S P T}\right)=E\left(\hat{\bar{Y}}_{S P T}-\bar{Y}\right)^{2}=\bar{Y}^{2} E\left(e_{0}-\theta_{1}^{\prime} e_{1}-\theta_{2}^{\prime} e_{2}\right)^{2} \\
& \Rightarrow \operatorname{MSE}\left(\hat{\bar{Y}}_{S P T}\right)=\bar{Y}^{2} E\left(e_{0}^{2}+\theta_{1}^{\prime 2} e_{1}^{2}+\theta_{2}^{\prime 2} e_{2}^{2}-2 \theta_{1}^{\prime} e_{0} e_{1}-2 \theta_{2}^{\prime} e_{0} e_{2}+2 \theta_{1}^{\prime} \theta_{2}^{\prime} e_{1} e_{2}\right) \\
& \Rightarrow \operatorname{MSE}\left(\hat{\bar{Y}}_{S P T}\right)=\bar{Y}^{2}\left\{E\left(e_{0}^{2}\right)+\theta_{1}^{\prime 2} E\left(e_{1}^{2}\right)+\theta_{2}^{\prime 2} E\left(e_{2}^{2}\right)-2 \theta_{1}^{\prime} E\left(e_{0} e_{1}\right)-2 \theta_{1}^{\prime} E\left(e_{0} e_{2}\right)+2 \theta_{1}^{\prime} \theta_{2}^{\prime} E\left(e_{1} e_{2}\right)\right\} \\
& \operatorname{MSE}\left(\hat{\bar{Y}}_{S P T}\right)=\frac{1-f}{n} \bar{Y}^{2}\left\{C_{y}^{2}+\theta_{1}^{\prime 2} C_{x_{1}}^{2}+\theta_{2}^{\prime 2} C_{x_{2}}^{2}-2 \theta_{1}^{\prime} \rho_{P x_{1}} C_{y} C_{x_{1}}-2 \theta_{2}^{\prime} \rho_{x x_{2}} C_{y} C_{x_{2}}+2 \theta_{1}^{\prime} \theta_{2}^{\prime} \rho_{x_{x_{2}}} C_{x_{x_{1}}} C_{x_{x_{2}}}\right\}
\end{aligned}
$$

The proposed modified ratio estimator $\hat{\bar{Y}}_{S P T}$ can be easily generalized to include several auxiliary variables. If $X_{1}, X_{2}, \ldots, X_{\mathrm{k}}$ are $k$ auxiliary variables that are positively correlated with a study variable $Y$, then the generalized modified ratio estimator is defined as

$$
\hat{\bar{Y}}_{G S P T}=\bar{y}\left(\frac{\alpha_{1}\left[\bar{X}_{1}+T_{1}\right]+\alpha_{2}\left[\bar{X}_{2}+T_{2}\right]+\alpha_{3}\left[\bar{X}_{3}+T_{3}\right]+\ldots+\alpha_{k}\left[\bar{X}_{k}+T_{k}\right]}{\alpha_{1}\left[\bar{X}_{1}+T_{1}\right]+\alpha_{2}\left[\bar{X}_{2}+T_{2}\right]+\alpha_{3}\left[\bar{X}_{3}+T_{3}\right]+\ldots+\alpha_{k}\left[\bar{X}_{k}+T_{k}\right]}\right)
$$

where $\alpha_{1}, \alpha_{2}, \ldots, \alpha_{k}$ are the weights and the $T_{1}, T_{2}, \ldots, T_{k}$ are the known parameters of the auxiliary variables.

\section{Efficiency Comparisons}

The efficiencies of the proposed estimators for estimating the finite population mean are assessed with that of SRSWOR sample mean and other existing estimators, as previously proposed.

From expressions (20) and (1), the proposed estimators $\hat{\bar{Y}}_{S P T}$ are more efficient than the SRSWOR sample mean $\bar{y}_{r}$. The derived conditions are: 


\section{SUBRAMANI \& PRABAVATHY}

$$
\operatorname{MSE}\left(\hat{\bar{Y}}_{S P T}\right) \leq V\left(\bar{y}_{r}\right)
$$

if

$$
\theta_{1}^{\prime 2} C_{x_{1}}^{2}+\theta_{2}^{\prime 2} C_{x_{2}}^{2} \leq 2\left(\theta_{1}^{\prime} \rho_{y x_{1}} C_{y} C_{x_{1}}+\theta_{2}^{\prime} \rho_{y x_{2}} C_{y} C_{x_{2}}-\theta_{1}^{\prime} \theta_{2}^{\prime} \rho_{x_{1} x_{2}} C_{x_{1}} C_{x_{2}}\right)
$$

From expressions (20) and (5), the proposed estimators $\hat{\bar{Y}}_{S P T}$ are more efficient than the existing ratio estimator $\hat{\bar{Y}}_{1}$. The derived conditions are:

$$
\operatorname{MSE}\left(\hat{\bar{Y}}_{S P T}\right) \leq \operatorname{MSE}\left(\hat{\bar{Y}}_{1}\right)
$$

if

$$
\left(\theta_{1}^{2}-1\right) C_{x_{1}}^{2}+\left(\theta_{2}^{\prime 2}-1\right) C_{x_{2}}^{2} \leq 2\left\{\left(\theta_{1}^{\prime}-1\right) \rho_{y x_{1}} C_{y} C_{x_{1}}+\left(\theta_{2}^{\prime}-1\right) \rho_{y x_{2}} C_{y} C_{x_{2}}-\left(\theta_{1}^{\prime} \theta_{2}^{\prime}-1\right) \rho_{x_{1} x_{2}} C_{x_{1}} C_{x_{2}}\right\}
$$

From expressions (20) and (7), the proposed estimators $\hat{\bar{Y}}_{S P T}$ are more efficient than the existing ratio estimator $\hat{\bar{Y}}_{2}$. The derived conditions are:

$$
\operatorname{MSE}\left(\hat{\bar{Y}}_{S P T}\right) \leq \operatorname{MSE}\left(\hat{\bar{Y}}_{2}\right)
$$

if

$$
\begin{aligned}
& \left(\theta_{1}^{2}-\mu_{1}^{* 2}\right) C_{x_{1}}^{2}+\left(\theta_{1}^{2}-\mu_{2}^{* 2}\right) C_{x_{2}}^{2} \leq \\
& 2\left\{\left(\theta_{1}^{\prime}-\mu_{1}^{*}\right) \rho_{y x_{1}} C_{y} C_{x_{1}}+\left(\theta_{2}^{\prime}-\mu_{2}^{*}\right) \rho_{y x_{2}} C_{y} C_{x_{2}}-\left(\theta_{1}^{\prime} \theta_{2}^{\prime}+\mu_{1}^{*} \mu_{2}^{*}\right) \rho_{x_{1} x_{2}} C_{x_{1}} C_{x_{2}}\right\}
\end{aligned}
$$

From expressions (20) and (9), the proposed estimators $\hat{\bar{Y}}_{S P T}$ are more efficient than the existing ratio estimator $\hat{\bar{Y}}_{3}$. The derived conditions are:

$$
\operatorname{MSE}\left(\hat{\bar{Y}}_{S P T}\right) \leq \operatorname{MSE}\left(\hat{\bar{Y}}_{3}\right)
$$

if

$$
\begin{aligned}
& \alpha_{1}^{2}\left(R_{s p}^{\prime 2}-R_{1}^{2}\right) S_{x_{1}}^{2}+\alpha_{2}^{2}\left(R_{s p}^{\prime 2}-R_{2}^{2}\right) S_{x_{2}}^{2}+B_{1} S_{y x_{1}}+B_{2} S_{y x_{2}} \leq \\
& 2\left\{R_{s p}^{\prime}\left(\alpha_{1} S_{y x_{1}}+\alpha_{2} S_{y x_{2}}\right)-\left[\alpha_{1} \alpha_{2} R_{s p}^{\prime 2}-\left(\alpha_{1} R_{1}+B_{1}\right)\left(\alpha_{2} R_{2}+B_{2}\right)\right] S_{x_{1} x_{2}}\right\}
\end{aligned}
$$




\section{TWO PARAMETER MODIFIED RATIO ESTIMATORS}

From expressions (20) and (11), the proposed estimators $\hat{\bar{Y}}_{S P T}$ are more efficient than the existing ratio estimator $\hat{\bar{Y}}_{4}$. The derived conditions are:

$$
\operatorname{MSE}\left(\hat{\bar{Y}}_{S P T}\right) \leq \operatorname{MSE}\left(\hat{\bar{Y}}_{4}\right)
$$

if

$$
\begin{aligned}
& {\left[\alpha_{1}^{2} R_{s p}^{\prime 2}-\left(1-\alpha_{1}\right)^{2} R_{1}^{2}\right] S_{x_{1}}^{2}+\left[\alpha_{2}^{2} R_{s p}^{\prime 2}-\left(1-\alpha_{2}\right)^{2} R_{2}^{2}\right] S_{x_{2}}^{2} \leq} \\
& \begin{aligned}
2\left\{S_{y x_{1}}\left[\alpha_{1} R_{s p}^{\prime}-\left(1-\alpha_{1}\right) R_{1}\right]+S_{y x_{2}}\right. & {\left[\alpha_{2} R_{s p}^{\prime}+\left(1-\alpha_{2}\right) R_{2}\right] } \\
& \left.-S_{x_{1} x_{2}}\left[\alpha_{1} \alpha_{2} R_{s p}^{\prime 2}+R_{1} R_{2}\left(1-\alpha_{1}\right)\left(1-\alpha_{2}\right)\right]\right\}
\end{aligned}
\end{aligned}
$$

From expressions (20) and (12), the proposed estimators $\hat{\bar{Y}}_{S P T}$ are more efficient than the existing ratio estimator $\hat{\bar{Y}}_{5}$. The derived conditions are:

$$
\operatorname{MSE}\left(\hat{\bar{Y}}_{S P T}\right) \leq \operatorname{MSE}\left(\hat{\bar{Y}}_{5}\right)
$$

if

$$
\begin{aligned}
& {\left[\alpha_{1}^{2} R_{s p}^{\prime 2}-\left(1-\alpha_{1}\right)^{2} R_{1}^{2}\right] S_{x_{1}}^{2}+\left[\alpha_{2}^{2} R_{s p}^{\prime 2}-\left(1-\alpha_{2}\right)^{2} R_{2}^{2}\right] S_{x_{2}}{ }^{2} \leq } \\
& 2\left\{S_{y x_{1}}\left[\alpha_{1} R_{s p}^{\prime}-\left(1-\alpha_{1}\right) R_{1}\right]\right.+S_{y x_{2}}\left[\alpha_{2} R_{s p}^{\prime}-\left(1-\alpha_{2}\right) R_{2}\right] \\
&-\left.S_{x_{1} x_{2}}\left[\alpha_{1} \alpha_{2} R_{s p}^{\prime 2}-R_{1} R_{2}\left(1-\alpha_{1}\right)\left(1-\alpha_{2}\right)\right]\right\}
\end{aligned}
$$

From expressions (20) and (13), the proposed estimators $\hat{\bar{Y}}_{S P T}$ are more efficient than the existing ratio estimator $\hat{\bar{Y}}_{6}$. The derived conditions are:

$$
\operatorname{MSE}\left(\hat{\bar{Y}}_{S P T}\right) \leq \operatorname{MSE}\left(\hat{\bar{Y}}_{6}\right)
$$

if

$$
\begin{aligned}
& {\left[\alpha_{1}^{2} R_{s p}^{\prime 2}-\left(1-\alpha_{1}\right)^{2} R_{1}^{2}\right] S_{x_{1}}^{2}+\left[\alpha_{2}^{2} R_{s p}^{\prime 2}-\left(1-\alpha_{2}\right)^{2} R_{2}^{2}\right] S_{x_{2}}^{2} \leq } \\
& 2\left\{S_{y x_{1}}\left[\alpha_{1} R_{s p}^{\prime}+\left(1-\alpha_{1}\right) R_{1}\right]\right.+S_{y x_{2}}\left[\alpha_{2} R_{s p}^{\prime}-\left(1-\alpha_{2}\right) R_{2}\right] \\
&-\left.S_{x_{1} x_{2}}\left[\alpha_{1} \alpha_{2} R_{s p}^{\prime 2}+R_{1} R_{2}\left(1-\alpha_{1}\right)\left(1-\alpha_{2}\right)\right]\right\}
\end{aligned}
$$




\section{SUBRAMANI \& PRABAVATHY}

where $R_{s p}^{\prime}=\frac{\bar{Y}}{\alpha_{1}\left(\bar{X}_{1}+T_{1}\right)+\alpha_{2}\left(\bar{X}_{2}+T_{2}\right)}$

\section{Numerical Study}

The performance of the proposed two parameter modified ratio estimators have been compared with that of the SRSWOR sample mean and some existing modified ratio estimators algebraically. However, the proposed estimators perform well compared to the existing estimators only under certain conditions and - for numerical comparisons - they are assessed for certain natural populations. In this connection, two natural populations were considered to assess the performance of the proposed estimators with that of existing estimators. Population 1 is from Singh and Chaudhary (1986, p. 177) and population 2 is from Kadilar and Cingi $(2009$, p. 117). The description of the study and auxiliary variables for the two populations are shown in Table 1.

Table 1. Description of the study variable and auxiliary variable

\begin{tabular}{clll} 
Population & Study Variable $\boldsymbol{Y}$ & Auxiliary Variable $\boldsymbol{X}_{\mathbf{1}}$ & Auxiliary Variable $\boldsymbol{X}_{\mathbf{2}}$ \\
\hline 1 & Area under wheat in 1974 & Area under wheat in1971 & Area under wheat in1973 \\
2 & Length of the fish & Length of the head & Length of the fin \\
\hline
\end{tabular}

The population parameters and constants computed for the two populations are given in Tables 2-4. 


\section{TWO PARAMETER MODIFIED RATIO ESTIMATORS}

Table 2. Parameters and Constants of the Populations

\begin{tabular}{lrrrrrrrrr} 
Parameter & $N$ & $n$ & $\bar{Y}$ & $\bar{X}_{1}$ & $\bar{X}_{2}$ & $\rho_{y x_{1}}$ & $\rho_{y x_{2}}$ & $\rho_{x_{1} x_{2}}$ & $\beta_{11}$ \\
\hline Pop. 1 & 34.00 & 20.00 & 856.41 & 208.88 & 199.44 & 0.45 & 0.45 & 0.98 & 0.87 \\
Pop. 2 & 25.00 & 10.00 & 75.28 & 14.30 & 6.82 & 0.99 & 0.89 & 0.92 & 1.24 \\
\hline & & & & & & & & & \\
Parameter & $\beta_{12}$ & $\beta_{21}$ & $\beta_{22}$ & $S_{y}$ & $C_{y}$ & $S_{x_{1}}$ & $S_{x_{2}}$ & $C_{x_{1}}$ & $C_{x_{2}}$ \\
\hline Pop. 1 & 1.28 & 2.91 & 3.73 & 733.14 & 0.86 & 150.51 & 150.22 & 0.72 & 0.75 \\
Pop. 2 & 0.86 & 4.26 & 4.35 & 17.27 & 0.23 & 3.17 & 1.53 & 0.22 & 0.22 \\
\hline
\end{tabular}

Table 3. Variance/Mean squared error of the existing and proposed estimators for Population 1

\begin{tabular}{|c|c|c|c|c|c|c|c|c|c|c|}
\hline \multicolumn{6}{|c|}{ Existing Estimators } & & & & & \\
\hline \multicolumn{3}{|c|}{$\hat{\bar{Y}}_{r}$} & $\hat{\bar{Y}}_{1}$ & \multicolumn{2}{|r|}{$\hat{\bar{Y}}_{2}$} & & & & & \\
\hline \multicolumn{3}{|c|}{37940.84} & 90847.02 & \multicolumn{2}{|c|}{40145.19} & \multicolumn{5}{|c|}{ Proposed Estimators } \\
\hline$\alpha_{1}$ & $\alpha_{2}$ & $\hat{\hat{Y}_{3}}$ & $\hat{\bar{Y}}_{4}$ & $\hat{\bar{Y}}_{5}$ & $\hat{\bar{Y}}_{6}$ & $\hat{\bar{Y}}_{S P 1}$ & $\hat{\bar{Y}}_{S P 2}$ & $\hat{\bar{Y}}_{S P 3}$ & $\hat{\bar{Y}}_{S P 4}$ & $\hat{\bar{Y}}_{S P 5}$ \\
\hline 0.0 & 1.0 & 67310.24 & 64818.97 & 64818.97 & 64818.97 & 37057.66 & 37047.45 & 37541.57 & 37466.93 & 37396.04 \\
\hline 0.1 & 0.9 & 62385.73 & 60005.70 & 60005.90 & 60005.94 & 36843.39 & 36834.06 & 37275.19 & 37210.98 & 37138.09 \\
\hline 0.2 & 0.8 & 58048.59 & 56317.41 & 56317.77 & 56317.84 & 36654.14 & 36645.64 & 37036.69 & 36982.32 & 36907.47 \\
\hline 0.3 & 0.7 & 54298.80 & 53754.11 & 53754.56 & 53754.66 & 36489.42 & 36481.71 & 36825.28 & 36780.23 & 36703.44 \\
\hline 0.4 & 0.6 & 51136.38 & 52315.78 & 52316.28 & 52316.42 & 36348.74 & 36341.77 & 36640.21 & 36604.01 & 36525.27 \\
\hline 0.5 & 0.5 & 48561.32 & 52002.43 & 52002.92 & 52003.10 & 36231.61 & 36225.37 & 36480.75 & 36452.97 & 36372.28 \\
\hline 0.6 & 0.4 & 46573.62 & 52814.07 & 52814.49 & 52814.70 & 36137.58 & 36132.02 & 36346.17 & 36326.46 & 36243.79 \\
\hline 0.7 & 0.3 & 45173.28 & 54750.68 & 54750.99 & 54751.23 & 36066.20 & 36061.30 & 36235.78 & 36223.84 & 36139.12 \\
\hline 0.8 & 0.2 & 44360.30 & 57812.27 & 57812.42 & 57812.69 & 36017.00 & 36012.74 & 36148.92 & 36144.46 & 36057.65 \\
\hline 0.9 & 0.1 & 44134.68 & 61998.84 & 61998.77 & 61999.08 & 35989.55 & 35985.91 & 36084.91 & 36087.73 & 35998.74 \\
\hline 1.0 & 0.0 & 44496.43 & 67310.39 & 67310.05 & 67310.39 & 35983.42 & 35980.39 & 36043.13 & 36053.04 & 35961.79 \\
\hline
\end{tabular}




\section{SUBRAMANI \& PRABAVATHY}

Table 4. Variance/Mean squared error of the existing and proposed estimators for

Population 2

\begin{tabular}{|c|c|c|c|c|c|c|c|c|c|c|}
\hline \multicolumn{6}{|c|}{ Existing Estimators } & & & & & \\
\hline & & & $\hat{\bar{Y}}_{1}$ & & $\hat{\bar{V}}_{2}$ & \multirow{2}{*}{\multicolumn{5}{|c|}{ Proposed Estimators }} \\
\hline \multicolumn{3}{|c|}{17.90} & 17.58 & \multicolumn{2}{|c|}{17.58} & & & & & \\
\hline$\alpha_{1}$ & $\alpha_{2}$ & $\hat{\overline{Y_{3}}}$ & $\hat{\bar{Y}}_{4}$ & $\hat{\bar{Y}}_{5}$ & $\hat{\bar{Y}}_{6}$ & $\hat{\bar{Y}}_{S P 1}$ & $\hat{\bar{Y}}_{S P 2}$ & $\hat{\bar{Y}}_{S P 3}$ & $\hat{\bar{Y}}_{S P 4}$ & $\hat{\bar{Y}}_{S P 5}$ \\
\hline 0.0 & 1.0 & 35.07 & 34.61 & 34.61 & 34.61 & 5.32 & 5.54 & 3.89 & 3.90 & 5.72 \\
\hline 0.1 & 0.9 & 32.15 & 31.58 & 31.62 & 31.64 & 4.50 & 4.72 & 2.84 & 2.84 & 4.72 \\
\hline 0.2 & 0.8 & 29.57 & 29.24 & 29.31 & 29.34 & 3.85 & 4.07 & 2.12 & 2.12 & 3.92 \\
\hline 0.3 & 0.7 & 27.33 & 27.58 & 27.67 & 27.71 & 3.32 & 3.53 & 1.62 & 1.62 & 3.28 \\
\hline 0.4 & 0.6 & 25.42 & 26.60 & 26.71 & 26.75 & 2.89 & 3.10 & 1.26 & 1.26 & 2.77 \\
\hline 0.5 & 0.5 & 23.85 & 26.31 & 26.41 & 26.47 & 2.54 & 2.74 & 1.01 & 1.01 & 2.36 \\
\hline 0.6 & 0.4 & 22.62 & 26.71 & 26.79 & 26.86 & 2.26 & 2.44 & 0.83 & 0.83 & 2.03 \\
\hline 0.7 & 0.3 & 21.72 & 27.78 & 27.83 & 27.92 & 2.02 & 2.19 & 0.70 & 0.70 & 1.76 \\
\hline 0.8 & 0.2 & 21.16 & 29.55 & 29.55 & 29.65 & 1.83 & 1.99 & 0.61 & 0.61 & 1.55 \\
\hline 0.9 & 0.1 & 20.94 & 31.99 & 31.94 & 32.05 & 1.67 & 1.81 & 0.55 & 0.55 & 1.38 \\
\hline 1.0 & 0.0 & 21.05 & 35.12 & 35.00 & 35.12 & 1.53 & 1.67 & 0.51 & 0.51 & 1.25 \\
\hline
\end{tabular}

From the values in Tables 3 and 4, the mean squared error of the proposed modified ratio estimators $\hat{\bar{Y}}_{S P j} j=1,2,3,4,5$ are less than the variance of SRSWOR sample mean, the mean squared error of the existing modified ratio estimators $\hat{\bar{Y}}_{j} ; j=1,2,3, \ldots, 6$. Further, to show the efficiency of the proposed estimators, the percentage relative efficiencies (PRE's) of the proposed estimators with respect to the existing estimators is computed by:

$$
\operatorname{PRE}\left(\hat{\bar{Y}}_{S P j}\right)=\frac{\operatorname{MSE}(.)}{\operatorname{MSE}\left(\hat{\bar{Y}}_{S P j}\right)} * 100 \text {. }
$$




\section{TWO PARAMETER MODIFIED RATIO ESTIMATORS}

Table 5. PRE of the proposed estimator $\hat{\bar{Y}}_{S P j}$ for Population 1

\begin{tabular}{|c|c|c|c|c|c|c|c|c|c|}
\hline \multirow{3}{*}{$\alpha_{1}$} & \multirow{3}{*}{$\alpha_{2}$} & \multirow{3}{*}{$\begin{array}{l}\text { Proposed } \\
\text { Estimators }\end{array}$} & \multicolumn{7}{|c|}{ Existing Estimators } \\
\hline & & & \multirow{2}{*}{$\begin{array}{c}\text { SRSWOR } \\
\bar{y}_{r}\end{array}$} & \multicolumn{6}{|c|}{ Modified Ratio Estimators } \\
\hline & & & & $\hat{\bar{Y}}_{1}$ & $\hat{\bar{Y}}_{2}$ & $\hat{\bar{Y}}_{3}$ & $\hat{\bar{Y}}_{4}$ & $\hat{\bar{Y}}_{5}$ & $\hat{\bar{Y}}_{6}$ \\
\hline \multirow{5}{*}{0.0} & & $\hat{\bar{Y}}_{S P 1}$ & 102.38 & 245.15 & 108.33 & 181.64 & 174.91 & 174.91 & 174.91 \\
\hline & & $\hat{\bar{Y}}_{S P 2}$ & 102.41 & 245.22 & 108.36 & 181.69 & 174.96 & 174.96 & 174.96 \\
\hline & 1.0 & $\hat{\bar{Y}}_{S P 3}$ & 101.06 & 241.99 & 106.94 & 179.30 & 172.66 & 172.66 & 172.66 \\
\hline & & $\hat{\bar{Y}}_{S P 4}$ & 101.26 & 242.47 & 107.15 & 179.65 & 173.00 & 173.00 & 173.00 \\
\hline & & $\hat{\bar{Y}}_{S P 5}$ & 101.46 & 242.93 & 107.35 & 179.99 & 173.33 & 173.33 & 173.33 \\
\hline \multirow{5}{*}{0.1} & & $\hat{\bar{Y}}_{S P 1}$ & 102.98 & 246.58 & 108.96 & 169.33 & 162.87 & 162.87 & 162.87 \\
\hline & & $\hat{\bar{Y}}_{S P 2}$ & 103.00 & 246.64 & 108.99 & 169.37 & 162.91 & 162.91 & 162.91 \\
\hline & 0.9 & $\hat{\bar{Y}}_{S P 3}$ & 101.79 & 243.72 & 107.70 & 167.37 & 160.98 & 160.98 & 160.98 \\
\hline & & $\hat{\bar{Y}}_{S P 4}$ & 101.96 & 244.14 & 107.89 & 167.65 & 161.26 & 161.26 & 161.26 \\
\hline & & $\hat{\bar{Y}}_{S P 5}$ & 102.16 & 244.62 & 108.10 & 167.98 & 161.57 & 161.58 & 161.58 \\
\hline \multirow{5}{*}{0.2} & & $\hat{\bar{Y}}_{S P 1}$ & 103.51 & 247.85 & 109.52 & 158.37 & 153.65 & 153.65 & 153.65 \\
\hline & & $\hat{\bar{Y}}_{S P 2}$ & 103.53 & 247.91 & 109.55 & 158.41 & 153.68 & 153.68 & 153.68 \\
\hline & 0.8 & $\hat{\bar{Y}}_{S P 3}$ & 102.44 & 245.29 & 108.39 & 156.73 & 152.06 & 152.06 & 152.06 \\
\hline & & $\hat{\bar{Y}}_{S P 4}$ & 102.59 & 245.65 & 108.55 & 156.96 & 152.28 & 152.28 & 152.28 \\
\hline & & $\hat{\bar{Y}}_{S P 5}$ & 102.80 & 246.15 & 108.77 & 157.28 & 152.59 & 152.59 & 152.59 \\
\hline \multirow{5}{*}{0.3} & & $\hat{\bar{Y}}_{S P 1}$ & 103.98 & 248.97 & 110.02 & 148.81 & 147.31 & 147.32 & 147.32 \\
\hline & & $\hat{\bar{Y}}_{S P 2}$ & 104.00 & 249.02 & 110.04 & 148.84 & 147.35 & 147.35 & 147.35 \\
\hline & 0.7 & $\hat{\bar{Y}}_{S P 3}$ & 103.03 & 246.70 & 109.02 & 147.45 & 145.97 & 145.97 & 145.97 \\
\hline & & $\hat{\bar{Y}}_{S P 4}$ & 103.16 & 247.00 & 109.15 & 147.63 & 146.15 & 146.15 & 146.15 \\
\hline & & $\hat{\bar{Y}}_{S P 5}$ & 103.37 & 247.52 & 109.38 & 147.94 & 146.46 & 146.46 & 146.46 \\
\hline
\end{tabular}




\section{SUBRAMANI \& PRABAVATHY}

Table 5, continued

\begin{tabular}{|c|c|c|c|c|c|c|c|c|c|}
\hline \multirow{3}{*}{$\alpha_{1}$} & \multirow{3}{*}{$\alpha_{2}$} & \multirow{3}{*}{$\begin{array}{l}\text { Proposed } \\
\text { Estimators }\end{array}$} & \multicolumn{7}{|c|}{ Existing Estimators } \\
\hline & & & \multirow{2}{*}{$\begin{array}{c}\text { SRSWOR } \\
\bar{y}_{r}\end{array}$} & \multicolumn{6}{|c|}{ Modified Ratio Estimators } \\
\hline & & & & $\hat{\bar{Y}}_{1}$ & $\hat{\bar{Y}}_{2}$ & $\hat{\bar{Y}}_{3}$ & $\hat{\bar{Y}}_{4}$ & $\hat{\bar{Y}}_{5}$ & $\hat{\bar{Y}}_{6}$ \\
\hline \multirow{5}{*}{0.4} & & $\hat{\bar{Y}}_{S P 1}$ & 104.38 & 249.93 & 110.44 & 140.68 & 143.93 & 143.93 & 143.93 \\
\hline & & $\hat{\bar{Y}}_{S P 2}$ & 104.40 & 249.98 & 110.47 & 140.71 & 143.95 & 143.96 & 143.96 \\
\hline & 0.6 & $\hat{\bar{Y}}_{S P 3}$ & 103.55 & 247.94 & 109.57 & 139.56 & 142.78 & 142.78 & 142.78 \\
\hline & & $\hat{\bar{Y}}_{S P 4}$ & 103.65 & 248.19 & 109.67 & 139.70 & 142.92 & 142.92 & 142.93 \\
\hline & & $\hat{\bar{Y}}_{S P 5}$ & 103.88 & 248.72 & 109.91 & 140.00 & 143.23 & 143.23 & 143.23 \\
\hline \multirow{5}{*}{0.5} & & $\hat{\bar{Y}}_{S P 1}$ & 104.72 & 250.74 & 110.80 & 134.03 & 143.53 & 143.53 & 143.53 \\
\hline & & $\hat{\bar{Y}}_{S P 2}$ & 104.74 & 250.78 & 110.82 & 134.05 & 143.55 & 143.55 & 143.55 \\
\hline & 0.5 & $\hat{\bar{Y}}_{S P 3}$ & 104.00 & 249.03 & 110.04 & 133.11 & 142.55 & 142.55 & 142.55 \\
\hline & & $\hat{\bar{Y}}_{S P 4}$ & 104.08 & 249.22 & 110.13 & 133.22 & 142.66 & 142.66 & 142.66 \\
\hline & & $\hat{\bar{Y}}_{S P 5}$ & 104.31 & 249.77 & 110.37 & 133.51 & 142.97 & 142.97 & 142.97 \\
\hline \multirow{5}{*}{0.6} & & $\hat{\bar{Y}}_{S P 1}$ & 104.99 & 251.39 & 111.09 & 128.88 & 146.15 & 146.15 & 146.15 \\
\hline & & $\hat{\bar{Y}}_{S P 2}$ & 105.01 & 251.43 & 111.11 & 128.90 & 146.17 & 146.17 & 146.17 \\
\hline & 0.4 & $\hat{\bar{Y}}_{S P 3}$ & 104.39 & 249.95 & 110.45 & 128.14 & 145.31 & 145.31 & 145.31 \\
\hline & & $\hat{\bar{Y}}_{S P 4}$ & 104.44 & 250.08 & 110.51 & 128.21 & 145.39 & 145.39 & 145.39 \\
\hline & & $\hat{\bar{Y}}_{S P 5}$ & 104.68 & 250.66 & 110.76 & 128.50 & 145.72 & 145.72 & 145.72 \\
\hline \multirow{5}{*}{0.7} & & $\hat{\bar{Y}}_{S P 1}$ & 104.99 & 251.38 & 111.09 & 125.00 & 151.50 & 151.50 & 151.50 \\
\hline & & $\hat{\bar{Y}}_{S P 2}$ & 104.74 & 250.79 & 110.83 & 124.71 & 151.15 & 151.15 & 151.15 \\
\hline & 0.3 & $\hat{\bar{Y}}_{S P 3}$ & 104.71 & 250.71 & 110.79 & 124.66 & 151.10 & 151.10 & 151.10 \\
\hline & & $\hat{\bar{Y}}_{S P 4}$ & 105.21 & 251.92 & 111.32 & 125.27 & 151.83 & 151.83 & 151.83 \\
\hline & & $\hat{\bar{Y}}_{S P 5}$ & 105.20 & 251.89 & 111.31 & 125.25 & 151.81 & 151.81 & 151.81 \\
\hline
\end{tabular}




\section{TWO PARAMETER MODIFIED RATIO ESTIMATORS}

Table 5, continued

\begin{tabular}{|c|c|c|c|c|c|c|c|c|c|}
\hline \multirow{3}{*}{$\alpha_{1}$} & \multirow{3}{*}{$\alpha_{2}$} & \multirow{3}{*}{$\begin{array}{l}\text { Proposed } \\
\text { Estimators }\end{array}$} & \multicolumn{7}{|c|}{ Existing Estimators } \\
\hline & & & \multirow{2}{*}{$\begin{array}{c}\text { SRSWOR } \\
\bar{y}_{r}\end{array}$} & \multicolumn{6}{|c|}{ Modified Ratio Estimators } \\
\hline & & & & $\hat{\bar{Y}_{1}}$ & $\hat{\bar{Y}}_{2}$ & $\hat{\bar{Y}}_{3}$ & $\hat{\bar{Y}}_{4}$ & $\hat{\bar{Y}}_{5}$ & $\hat{\bar{Y}}_{6}$ \\
\hline \multirow{5}{*}{0.8} & & $\hat{\bar{Y}}_{S P 1}$ & 105.34 & 252.23 & 111.46 & 123.16 & 160.51 & 160.51 & 160.52 \\
\hline & & $\hat{\bar{Y}}_{S P 2}$ & 105.35 & 252.26 & 111.47 & 123.18 & 160.53 & 160.53 & 160.53 \\
\hline & 0.2 & $\hat{\bar{Y}}_{S P 3}$ & 104.96 & 251.31 & 111.06 & 122.72 & 159.93 & 159.93 & 159.93 \\
\hline & & $\hat{\bar{Y}}_{S P 4}$ & 104.97 & 251.34 & 111.07 & 122.73 & 159.95 & 159.95 & 159.95 \\
\hline & & $\hat{\bar{Y}}_{S P 5}$ & 105.22 & 251.95 & 111.34 & 123.03 & 160.33 & 160.33 & 160.33 \\
\hline \multirow{5}{*}{0.9} & & $\hat{\bar{Y}}_{S P 1}$ & 105.42 & 252.43 & 111.55 & 122.63 & 172.27 & 172.27 & 172.27 \\
\hline & & $\hat{\bar{Y}}_{S P 2}$ & 105.43 & 252.45 & 111.56 & 122.64 & 172.29 & 172.29 & 172.29 \\
\hline & 0.1 & $\hat{\bar{Y}}_{S P 3}$ & 105.14 & 251.76 & 111.25 & 122.31 & 171.81 & 171.81 & 171.81 \\
\hline & & $\hat{\bar{Y}}_{S P 4}$ & 105.14 & 251.74 & 111.24 & 122.30 & 171.80 & 171.80 & 171.80 \\
\hline & & $\hat{\bar{Y}}_{S P 5}$ & 105.39 & 252.36 & 111.52 & 122.60 & 172.23 & 172.22 & 172.23 \\
\hline \multirow{5}{*}{1.0} & & $\hat{\bar{Y}}_{S P 1}$ & 105.44 & 252.47 & 111.57 & 123.66 & 187.06 & 187.06 & 187.06 \\
\hline & & $\hat{\bar{Y}}_{S P 2}$ & 105.45 & 252.49 & 111.58 & 123.67 & 187.08 & 187.07 & 187.08 \\
\hline & 0.0 & $\hat{\bar{Y}}_{S P 3}$ & 105.27 & 252.05 & 111.38 & 123.45 & 186.75 & 186.75 & 186.75 \\
\hline & & $\hat{\bar{Y}}_{S P 4}$ & 105.24 & 251.98 & 111.35 & 123.42 & 186.70 & 186.70 & 186.70 \\
\hline & & $\hat{\bar{Y}}_{S P 5}$ & 105.50 & 252.62 & 111.63 & 123.73 & 187.17 & 187.17 & 187.17 \\
\hline
\end{tabular}




\section{SUBRAMANI \& PRABAVATHY}

Table 5 shows the following ranges for the PRE of the proposed estimators:

- from 101.06 to 105.50 in comparison with the SRSWOR sample mean;

- $\quad$ from 241.99 to 252.62 in comparison with the existing estimator $\hat{\bar{Y}}_{1}$ defined in (4);

- $\quad$ from 106.94 to 111.63 in comparison with the existing estimator $\hat{\bar{Y}}_{2}$ defined in (6);

- $\quad$ from 122.30 to 181.69 in comparison with the existing estimator $\hat{\bar{Y}}_{3}$ defined in (8);

- $\quad$ from 142.55 to 187.17 in comparison with the existing estimator $\hat{\bar{Y}}_{4}$, $\hat{\bar{Y}}_{5}, \hat{\bar{Y}}_{6}$ defined in (10).

Based on these comparisons, it is concluded that the proposed estimators perform better than the SRSWOR sample mean and other existing ratio estimators for the natural population 1 considered in this study.

Table 6. PRE of the proposed estimator $\hat{\bar{Y}}_{S P j}$ for Population 2

\begin{tabular}{|c|c|c|c|c|c|c|c|c|c|}
\hline \multirow{3}{*}{$\boldsymbol{a}_{1}$} & \multirow{3}{*}{$\alpha_{2}$} & \multirow{3}{*}{$\begin{array}{l}\text { Proposed } \\
\text { Estimators }\end{array}$} & \multicolumn{7}{|c|}{ Existing Estimators } \\
\hline & & & \multirow{2}{*}{$\begin{array}{c}\text { SRSWOR } \\
\bar{y}_{r}\end{array}$} & \multicolumn{6}{|c|}{ Modified Ratio Estimators } \\
\hline & & & & $\hat{\bar{Y}}_{1}$ & $\hat{\bar{Y}}_{2}$ & $\hat{\overline{Y_{3}}}$ & $\hat{\bar{Y}}_{4}$ & $\hat{\overline{Y_{5}}}$ & $\hat{\bar{Y}}_{6}$ \\
\hline \multirow{5}{*}{0.0} & \multirow{5}{*}{1.0} & $\hat{\bar{Y}}_{S P 1}$ & 336.47 & 330.45 & 330.45 & 659.21 & 650.56 & 650.56 & 650.56 \\
\hline & & $\hat{\bar{Y}}_{S P 2}$ & 323.10 & 317.33 & 317.33 & 633.03 & 624.73 & 624.73 & 624.73 \\
\hline & & $\hat{\bar{Y}}_{S P 3}$ & 460.15 & 451.93 & 451.93 & 901.54 & 889.72 & 889.72 & 889.72 \\
\hline & & $\hat{\bar{Y}}_{S P 4}$ & 458.97 & 450.77 & 450.77 & 899.23 & 887.44 & 887.44 & 887.44 \\
\hline & & $\hat{\bar{Y}}_{S P 5}$ & 312.94 & 307.34 & 307.34 & 613.11 & 605.07 & 605.07 & 605.07 \\
\hline
\end{tabular}




\section{TWO PARAMETER MODIFIED RATIO ESTIMATORS}

Table 6, continued

\begin{tabular}{|c|c|c|c|c|c|c|c|c|c|}
\hline \multirow{3}{*}{$\boldsymbol{\alpha}_{1}$} & \multirow{3}{*}{$\alpha_{2}$} & \multirow{3}{*}{$\begin{array}{l}\text { Proposed } \\
\text { Estimators }\end{array}$} & \multicolumn{7}{|c|}{ Existing Estimators } \\
\hline & & & \multirow{2}{*}{$\begin{array}{c}\text { SRSWOR } \\
\bar{y}_{r}\end{array}$} & \multicolumn{6}{|c|}{ Modified Ratio Estimators } \\
\hline & & & & $\hat{\bar{Y}}_{1}$ & $\hat{\bar{Y}}_{2}$ & $\hat{\bar{Y}}_{3}$ & $\hat{\bar{Y}}_{4}$ & $\hat{\bar{Y}}_{5}$ & $\hat{\bar{Y}}_{6}$ \\
\hline \multirow{5}{*}{0.1} & \multirow{5}{*}{0.9} & $\hat{\bar{Y}}_{S P 1}$ & 397.78 & 390.67 & 390.67 & 714.44 & 701.78 & 702.67 & 703.11 \\
\hline & & $\hat{\bar{Y}}_{S P 2}$ & 379.24 & 372.46 & 372.46 & 681.14 & 669.07 & 669.92 & 670.34 \\
\hline & & $\hat{\bar{Y}}_{S P 3}$ & 630.28 & 619.01 & 619.01 & 1132.04 & 1111.97 & 1113.38 & 1114.08 \\
\hline & & $\hat{\bar{Y}}_{S P 4}$ & 630.28 & 619.01 & 619.01 & 1132.04 & 1111.97 & 1113.38 & 1114.08 \\
\hline & & $\hat{\bar{Y}}_{S P 5}$ & 379.24 & 372.46 & 372.46 & 681.14 & 669.07 & 669.92 & 670.34 \\
\hline \multirow{5}{*}{0.2} & \multirow{5}{*}{0.8} & $\hat{\bar{Y}}_{S P 1}$ & 464.94 & 456.62 & 456.62 & 768.05 & 759.48 & 761.30 & 762.08 \\
\hline & & $\hat{\bar{Y}}_{S P 2}$ & 439.80 & 431.94 & 431.94 & 726.54 & 718.43 & 720.15 & 720.88 \\
\hline & & $\hat{\bar{Y}}_{S P 3}$ & 844.34 & 829.25 & 829.25 & 1394.81 & 1379.25 & 1382.55 & 1383.96 \\
\hline & & $\hat{\bar{Y}}_{S P 4}$ & 844.34 & 829.25 & 829.25 & 1394.81 & 1379.25 & 1382.55 & 1383.96 \\
\hline & & $\hat{\bar{Y}}_{S P 5}$ & 456.63 & 448.47 & 448.47 & 754.34 & 745.92 & 747.70 & 748.47 \\
\hline \multirow{5}{*}{0.3} & \multirow{5}{*}{0.7} & $\hat{\bar{Y}}_{S P 1}$ & 539.16 & 529.52 & 529.52 & 823.19 & 830.72 & 833.43 & 834.64 \\
\hline & & $\hat{\bar{Y}}_{S P 2}$ & 507.08 & 498.02 & 498.02 & 774.22 & 781.30 & 783.85 & 784.99 \\
\hline & & $\hat{\bar{Y}}_{S P 3}$ & 1104.94 & 1085.19 & 1085.19 & 1687.04 & 1702.47 & 1708.02 & 1710.49 \\
\hline & & $\hat{\bar{Y}}_{S P 4}$ & 1104.94 & 1085.19 & 1085.19 & 1687.04 & 1702.47 & 1708.02 & 1710.49 \\
\hline & & $\hat{\bar{Y}}_{S P 5}$ & 545.73 & 535.98 & 535.98 & 833.23 & 840.85 & 843.60 & 844.82 \\
\hline \multirow{5}{*}{0.4} & \multirow{5}{*}{0.6} & $\hat{\bar{Y}}_{S P 1}$ & 619.38 & 608.30 & 608.30 & 879.58 & 920.42 & 924.22 & 925.61 \\
\hline & & $\hat{\bar{Y}}_{S P 2}$ & 577.42 & 567.10 & 567.10 & 820.00 & 858.06 & 861.61 & 862.90 \\
\hline & & $\hat{\bar{Y}}_{S P 3}$ & 1420.63 & 1395.24 & 1395.24 & 2017.46 & 2111.11 & 2119.84 & 2123.02 \\
\hline & & $\hat{\bar{Y}}_{S P 4}$ & 1420.63 & 1395.24 & 1395.24 & 2017.46 & 2111.11 & 2119.84 & 2123.02 \\
\hline & & $\hat{\bar{Y}}_{S P 5}$ & 646.21 & 634.66 & 634.66 & 917.69 & 960.29 & 964.26 & 965.70 \\
\hline
\end{tabular}




\section{SUBRAMANI \& PRABAVATHY}

Table 6, continued

\begin{tabular}{|c|c|c|c|c|c|c|c|c|c|}
\hline \multirow{3}{*}{$a_{1}$} & \multirow{3}{*}{$\alpha_{2}$} & \multirow{3}{*}{$\begin{array}{l}\text { Proposed } \\
\text { Estimators }\end{array}$} & \multicolumn{7}{|c|}{ Existing Estimators } \\
\hline & & & \multirow{2}{*}{$\begin{array}{c}\text { SRSWOR } \\
\bar{y}_{r}\end{array}$} & \multicolumn{6}{|c|}{ Modified Ratio Estimators } \\
\hline & & & & $\hat{\bar{Y}}_{1}$ & $\hat{\bar{Y}}_{2}$ & $\hat{\overline{Y_{3}}}$ & $\hat{\bar{Y}}_{4}$ & $\hat{\bar{Y}_{5}}$ & $\hat{\bar{Y}}_{6}$ \\
\hline \multirow{5}{*}{0.5} & \multirow{5}{*}{0.5} & $\hat{\bar{Y}}_{S P 1}$ & 704.72 & 692.13 & 692.13 & 938.98 & 1035.83 & 1039.76 & 1042.13 \\
\hline & & $\hat{\bar{Y}}_{S P 2}$ & 653.28 & 641.61 & 641.61 & 870.44 & 960.22 & 963.87 & 966.06 \\
\hline & & $\hat{\bar{Y}}_{S P 3}$ & 1772.28 & 1740.59 & 1740.59 & 2361.39 & 2604.95 & 2614.85 & 2620.79 \\
\hline & & $\hat{\bar{Y}}_{S P 4}$ & 2361.39 & 2604.95 & 2614.85 & 1772.28 & 1740.59 & 1740.59 & 1772.28 \\
\hline & & $\hat{\bar{Y}}_{S P 5}$ & 758.47 & 744.92 & 744.92 & 1010.59 & 1114.83 & 1119.07 & 1121.61 \\
\hline \multirow{5}{*}{0.6} & \multirow{5}{*}{0.4} & $\hat{\bar{Y}}_{S P 1}$ & 792.04 & 777.88 & 777.88 & 1000.88 & 1181.86 & 1185.40 & 1188.50 \\
\hline & & $\hat{\bar{Y}}_{S P 2}$ & 733.61 & 720.49 & 720.49 & 927.05 & 1094.67 & 1097.95 & 1100.82 \\
\hline & & $\hat{\bar{Y}}_{S P 3}$ & 2156.63 & 2118.07 & 2118.07 & 2725.30 & 3218.07 & 3227.71 & 3236.14 \\
\hline & & $\hat{\bar{Y}}_{S P 4}$ & 2156.63 & 2118.07 & 2118.07 & 2725.30 & 3218.07 & 3227.71 & 3236.14 \\
\hline & & $\hat{\bar{Y}}_{S P 5}$ & 881.77 & 866.01 & 866.01 & 1114.29 & 1315.76 & 1319.70 & 1323.15 \\
\hline \multirow{5}{*}{0.7} & \multirow{5}{*}{0.3} & $\hat{\bar{Y}}_{S P 1}$ & 886.14 & 870.30 & 870.30 & 1075.25 & 1375.25 & 1377.72 & 1382.18 \\
\hline & & $\hat{\bar{Y}}_{S P 2}$ & 817.35 & 802.74 & 802.74 & 991.78 & 1268.49 & 1270.78 & 1274.89 \\
\hline & & $\hat{\bar{Y}}_{S P 3}$ & 2557.14 & 2511.43 & 2511.43 & 3102.86 & 3968.57 & 3975.71 & 3988.57 \\
\hline & & $\hat{\bar{Y}}_{S P 4}$ & 2557.14 & 2511.43 & 2511.43 & 3102.86 & 3968.57 & 3975.71 & 3988.57 \\
\hline & & $\hat{\bar{Y}}_{S P 5}$ & 1017.05 & 998.86 & 998.86 & 1234.09 & 1578.41 & 1581.25 & 1586.36 \\
\hline \multirow{5}{*}{0.8} & \multirow{5}{*}{0.2} & $\hat{\bar{Y}}_{S P 1}$ & 978.14 & 960.66 & 960.66 & 1156.28 & 1614.75 & 1614.75 & 1620.22 \\
\hline & & $\hat{\bar{Y}}_{S P 2}$ & 899.50 & 883.42 & 883.42 & 1063.32 & 1484.92 & 1484.92 & 1489.95 \\
\hline & & $\hat{\bar{Y}}_{S P 3}$ & 2934.43 & 2881.97 & 2881.97 & 3468.85 & 4844.26 & 4844.26 & 4860.66 \\
\hline & & $\hat{\bar{Y}}_{S P 4}$ & 2934.43 & 2881.97 & 2881.97 & 3468.85 & 4844.26 & 4844.26 & 4860.66 \\
\hline & & $\hat{\bar{Y}}_{S P 5}$ & 1154.84 & 1134.19 & 1134.19 & 1365.16 & 1906.45 & 1906.45 & 1912.90 \\
\hline
\end{tabular}




\section{TWO PARAMETER MODIFIED RATIO ESTIMATORS}

Table 6, continued

\begin{tabular}{|c|c|c|c|c|c|c|c|c|c|}
\hline \multirow{3}{*}{$\alpha_{1}$} & \multirow{3}{*}{$\alpha_{2}$} & \multirow{3}{*}{$\begin{array}{l}\text { Proposed } \\
\text { Estimators }\end{array}$} & \multicolumn{7}{|c|}{ Existing Estimators } \\
\hline & & & \multirow{2}{*}{$\begin{array}{c}\text { SRSWOR } \\
\bar{y}_{r}\end{array}$} & \multicolumn{6}{|c|}{ Modified Ratio Estimators } \\
\hline & & & & $\hat{\overline{Y_{1}}}$ & $\hat{\bar{Y}}_{2}$ & $\hat{\overline{Y_{3}}}$ & $\hat{\bar{Y}}_{4}$ & $\hat{\bar{Y}}_{5}$ & $\hat{\bar{Y}}_{6}$ \\
\hline \multirow{5}{*}{0.9} & \multirow{5}{*}{0.1} & $\hat{\bar{Y}}_{S P 1}$ & 1071.86 & 1052.69 & 1052.69 & 1253.89 & 1915.57 & 1912.57 & 1919.16 \\
\hline & & $\hat{\bar{Y}}_{S P 2}$ & 988.95 & 971.27 & 971.27 & 1156.91 & 1767.40 & 1764.64 & 1770.72 \\
\hline & & $\hat{\bar{Y}}_{S P 3}$ & 3254.55 & 3196.36 & 3196.36 & 3807.27 & 5816.36 & 5807.27 & 5827.27 \\
\hline & & $\hat{\bar{Y}}_{S P 4}$ & 3254.55 & 3196.36 & 3196.36 & 3807.27 & 5816.36 & 5807.27 & 5827.27 \\
\hline & & $\hat{\bar{Y}}_{S P 5}$ & 1297.10 & 1273.91 & 1273.91 & 1517.39 & 2318.12 & 2314.49 & 2322.46 \\
\hline \multirow{5}{*}{1.0} & \multirow{5}{*}{0.0} & $\hat{\bar{Y}}_{S P 1}$ & 1169.93 & 1149.02 & 1149.02 & 1375.82 & 2295.42 & 2287.58 & 2295.42 \\
\hline & & $\hat{\bar{Y}}_{S P 2}$ & 1071.86 & 1052.69 & 1052.69 & 1260.48 & 2102.99 & 2095.81 & 2102.99 \\
\hline & & $\hat{\bar{Y}}_{S P 3}$ & 3509.80 & 3447.06 & 3447.06 & 4127.45 & 6886.27 & 6862.75 & 6886.27 \\
\hline & & $\hat{\bar{Y}}_{S P 4}$ & 3509.80 & 3447.06 & 3447.06 & 4127.45 & 6886.27 & 6862.75 & 6886.27 \\
\hline & & $\hat{\bar{Y}}_{S P 5}$ & 1432.00 & 1406.40 & 1406.40 & 1684.00 & 2809.60 & 2800.00 & 2809.60 \\
\hline
\end{tabular}

Table 6 shows the following ranges for the PRE of the proposed estimators:

- $\quad$ from 312.94 to 3509.80 in comparison with SRSWOR sample mean;

- $\quad$ from 307.34 to 3447.06 in comparison with the existing estimator $\hat{\bar{Y}}_{1}$ defined in (4) and $\hat{\bar{Y}}_{2}$ defined in (6);

- from 613.11 to 4127.45 in comparison with the existing estimator $\hat{\bar{Y}}_{3}$ defined in (8);

- $\quad$ from 605.07 to 6886.27 in comparison with the existing estimator $\hat{\bar{Y}}_{4}$ defined in (10);

- from 605.07 to 6862.75 in comparison with the existing estimator $\hat{\bar{Y}}_{5}$ defined in (10);

- $\quad$ from 605.07 to 6886.27 in comparison with the existing estimator $\hat{\bar{Y}}_{6}$ defined in (10). 


\section{SUBRAMANI \& PRABAVATHY}

Based on these comparisons, it may be concluded that the proposed estimators perform better than the SRSWOR sample mean and other existing ratio estimators for the natural population 2 considered in this study.

\section{Conclusion}

This article proposed two parameter modified ratio estimators with known correlation coefficient, skewness and kurtosis of the auxiliary variables and their linear combinations. The mean squared errors of the proposed estimators were derived and compared with that of SRSWOR sample mean, the classical ratio estimator and the existing modified ratio estimators. The performance of the proposed estimators was also assessed with that of the existing estimators for certain natural populations. It was observed from the numerical comparisons that the mean squared errors of the proposed estimators are less than the mean squared error of the existing estimators. Further it was shown that the PREs of the proposed estimators, with respect to existing estimators, range from 101.06 to 6886.27. Hence, the proposed modified ratio estimators are strongly recommended and may be preferred over existing estimators for practical applications.

\section{Acknowledgements}

The authors wish to extend their gratitude and thanks for the financial assistance received through UGC-Major Research Project, and to the Editor and the Referees for their effort to improve the presentation of the paper.

\section{References}

Abu-Dayyeh, W. A., Ahmed, M. S., Ahmed, R. A., \& Muttlak, H. A. (2003). Some estimators of finite population mean using auxiliary information. Applied Mathematics and Computation, 139: 287-298.

Bandyopadhyay, S. (1980). Improved ratio and product estimators. Sankhyā $C, 42: 45-49$.

Cochran, W. G. (1940). The estimation of the yields of cereal experiments by sampling for the ratio of grain to total produce. Journal of Agriculture Science, 37: 199-212. 


\section{TWO PARAMETER MODIFIED RATIO ESTIMATORS}

Cochran, W. G. (1977). Sampling techniques. New York, NY: John Wiley \& Sons.

Kadilar, C., \& Cingi, H. (2004). Estimator of a population mean using two auxiliary variables in simple random sampling. International Mathematical Journal, 5: 357-360.

Kadilar, C., \& Cingi, H. (2005). A new estimator using two auxiliary variables. Applied Mathematics and Computation, 162, 901-908.

Kadilar, C., \& Cingi, H. (2009). Advances in sampling theory-ratio method of estimation. Oak Park, IL: Bentham Science Publishers.

Khare, B. B., Srivastava, U., \& Kumar, K. (2013). A generalized chain ratio in regression estimator for population mean using two auxiliary characters in sample survey. Journal of Scientific Research, 57: 147-153.

Murthy, M. N. (1967). Sampling Theory and Methods. Calcutta, India: Statistical Publishing Society.

Naik, V. D., \& Gupta, P. C. (1991). A general class of estimators for estimating population mean using auxiliary information. Metrika, 38: 11-17.

Olkin, I. (1958). Multivariate ratio estimation for finite populations. Biometrika, 45: 154-165.

Perri, P. F. (2004). Alcune considerazioni sull'efficienza degli stimatori rapporto-cum-prodotto. Statistica \& Applicazioni, 2(2): 59-75.

Perri, P. F. (2007). Improved ratio-cum-product type estimators. Statistics in Transition-NS, 8(1): 51-69.

Raj, D. (1965). On a method of using multi-auxiliary information in sample surveys. Journal of the American Statistical Association, 60: 154-165.

Rao, P. S. R. S., \& Mudholkar, G. S. (1967). Generalized multivariate estimator for the mean of finite populations. Journal of the American Statistical Association, 62: 1009-1012.

Sahoo, L. N., \& Swain, A. K. P. C. (1980). Unbiased ratio-cum-product estimator. Sankhyā C, 42: 56-62.

Singh, D., \& Chaudhary, F. S. (1986). Theory and analysis of sample survey designs. New Delhi, India: New Age International Publisher.

Singh, G. N. (2003). On the improvement of product method of estimation in sample surveys. Journal of the Indian Society of Agricultural Statistics, 56(3): 267-265.

Singh, H. P., \& Tailor, R. (2003). Use of known correlation coefficient in estimating the finite population means. Statistics in Transition, 6(4): 555-560. 


\section{SUBRAMANI \& PRABAVATHY}

Singh, H. P., \& Tailor, R. (2005). Estimation of finite population mean using known correlation coefficient between auxiliary characters. Statistica, 65 : 407-418.

Singh, H. P., Tailor, R., \& Kakran, M. S. (2004). Improved estimators of population mean using power transformation. Journal of the Indian Society of Agricultural Statistics, 58(2): 223-230.

Singh, M. P. (1965). On the estimation of ratio and product of the population parameters. Sankhyā B, 27: 321-328.

Singh, M. P. (1967a). Multivariate product method of estimation for finite populations. Journal of the Indian Society of Agricultural Statistics, 31, 375-378.

Singh, M. P. (1967b). Ratio cum product method of estimation. Metrika, 12: 34-42.

Sisodia, B. V. S., \& Dwivedi, V. K. (1981). A modified ratio estimator using coefficient of variation of auxiliary variable. Journal of the Indian Society of Agricultural Statistics, 33(1):13-18.

Srivenkataramana, T. (1980). A dual to ratio estimator in sample surveys. Biometrika, 67: 199-204.

Srivenkataramana, T., \& Tracy, D. S. (1981). An alternative to ratio method in sample surveys. Annals of the Institute of Statistical Mathematics, 32: 111-120.

Subramani, J. (2013).Generalized Modified Ratio Estimator for Estimation of Finite Population Mean. Journal of Modern Applied Statistical Methods, 12(2), 121-155.

Subramani, J., \& Kumarapandiyan, G. (2012a). Estimation of population mean using known median and co-efficient of skewness. American Journal of Mathematics and Statistics, 2(5): 101-107.

Subramani, J., \& Kumarapandiyan, G. (2012b). Estimation of population mean using co-efficient of variation and median of an auxiliary variable. International Journal of Probability and Statistics, 1(4): 111-118.

Subramani, J., \& Kumarapandiyan, G. (2012c). Modified ratio estimators using known median and co-efficient of kurtosis, American Journal of Mathematics and Statistics, 2(4): 95-100.

Subramani, J., \& Kumarapandiyan, G. (2013). A new modified ratio estimator for estimation of population mean when median of the auxiliary variable is known. Pakistan Journal of Statistics and Operation Research, 9(2), 137-145. 


\section{TWO PARAMETER MODIFIED RATIO ESTIMATORS}

Tailor, R., Parmar, R., Kim, J. M., \& Tailor, R. (2011). Ratio-cum-Product estimators of population mean using known population parameters of auxiliary variable. Communication of the Korean Statistical Society, 18(2): 155-164.

Tracy, D. S., Singh, H. P., \& Singh, R. (1996). An alternative to the ratiocum- product estimator in sample surveys. Journal of Statistical Planning and Inference, 53: 375-387.

Upadhyaya, L. N., \& Singh, H. P. (1999). Use of transformed auxiliary variable in estimating the finite population mean. Biometrical Journal, 41(5): 627-636.

Yan, Z., \& Tian, B. (2010). Ratio method to the mean estimation using coefficient of skewness of auxiliary variable. ICICA 2010, Part II, CCIS 106: 103-110. 